\section{$\underset{\substack{\text { hommes } \\ \text { \& migrations }}}{ }$}

\section{Hommes \& migrations}

Revue française de référence sur les dynamiques

migratoires

1303 | 2013

Diasporas marocaines

\title{
Janus ou la logique de la calculette
}

\section{Mustapha Harzoune}

\section{(2) OpenEdition \\ Journals}

\section{Édition électronique}

URL : http://journals.openedition.org/hommesmigrations/2597

DOI : 10.4000/hommesmigrations.2597

ISSN : 2262-3353

\section{Éditeur}

Musée national de l'histoire de l'immigration

\section{Édition imprimée}

Date de publication : 1 juillet 2013

Pagination : 189-192

ISBN : 978-2-919040-23-0

ISSN : $1142-852 X$

\section{Référence électronique}

Mustapha Harzoune, « Janus ou la logique de la calculette », Hommes \& migrations [En ligne], 1303 |

2013, mis en ligne le 22 janvier 2014, consulté le 22 septembre 2020. URL : http://

journals.openedition.org/hommesmigrations/2597; DOl : https://doi.org/10.4000/

hommesmigrations. 2597 


\title{
KIOSQUE
}

\section{JANUS OU LA LOGIQUE DE LA CALCULETTE}

\author{
MUSTAPHA HARZOUNE
}

es États-Unis s'apprêteraient à régulariser 11 Lmillions de migrants quand l'Allemagne en a accueilli 1 million, la pêche mondialisée aux cerveaux étend ses filets au moment où sont loués l'esprit d'entreprise et le "potentiel" des diplômés étrangers. A contrario, I'horizon s'assombrit pour les immigrés en Grande-Bretagne, en Suède, en Suisse ou en Israël qui expulsent l'indésirable Africain. Les États et les opinions semblent tanguer entre deux politiques contraires. En apparence. En matière d'immigration, la logique de la calculette, électorale ou comptable, ne dicterait-elle pas sa loi aux deux faces - ouverture ou fermeture - de ce Janus politique ? Le décompte des petits intérêts repose sur des stratégies de courte vue en dépit de faits - et de prévisions - qui inscrivent les migrations dans le quotidien de chacun et le devenir planétaire de tous. Le 20 juin, le Sénat américain a adopté une proposition de loi pour réformer la politique migratoire des États-Unis. Ce texte de "compromis", où le volet "régularisation" de 11 millions de clandestins a pour pendant un volet "sécularisation" (Le Monde.fr, 24 juin), "tente de satisfaire à la fois les démocrates et les républicains. En échange d'un processus de treize ans au terme duquel les personnes en situation irrégulière pourront demander la naturalisation américaine, le plan prévoit le déploiement sans précédent de 20000 agents supplémentaires le long des $3200 \mathrm{~km}$ de frontière avec le Mexique, en plus des 18000 déjà en place. La quasi-totalité de la frontière terrestre sera bordée de clôtures, et un arsenal paramilitaire de surveillance (caméras, détecteurs de mouvement, radars) aidera les policiers. Des drones voleront jour et nuit" (20 Minutes, 28 juin).

\section{Quand Janus entrouvre les portes}

Si le Parti républicain amorce "une stratégie de reconquête politique des Latinos, population en plein essor qu'il s'était aliénée en prônant une politique de reconduction systématique des illégaux à la frontière", les "conservateurs purs et durs" de la Chambre "considèrent le plan sénatorial comme une amnistie pure et simple des illégaux, qui récompense la violation de la loi et accouchera de nouvelles vagues d'immigrants" - notamment les élus qui ne sont pas confrontés à la pression du vote latino et/ou proches des électeurs du Tea Party (Le Figaro, 28 juin).

"Le coût total de cet effort devrait sélever à 40 milliards de dollars" (Le Figaro, 28 juin). Pour le démocrate Chuck Schumer, un des architectes de la réforme, "c'est cher, mais c'est le prix pour rassurer les Américains". "C'est vraiment de la politique, et cela n'a rien à voir avec ce qui se passe sur le terrain, explique Doris Meissner, experte au Migration Policy Institute. Jamais le nombre de passages clandestins n'a été aussi bas depuis quarante ans, rappelle-t-elle, notamment grâce aux investissements réalisés depuis le 11 septembre 2001. Les auteurs du texte en conviennent: le républicain 


\section{KIOSQUE}

Bob Corker a lui-même admis qu'ils en avaient peut-être fait 'un peu trop'” (Le Point.fr, 25 juin). Pour le journaliste Jim Avila, "la loi pour réformer la politique migratoire a été détournée. D'une réforme ambitieuse, elle est devenue une loi sur la protection des frontières. (...) Alors que la présence des forces américaines diminue sur les anciennes zones de guerre, les entreprises d'armement et de défense voient dans la protection des frontières de nouveaux débouchés. À tel point que les défenseurs des libertés civiles s'in-

Selon une enquête de l'Ifop,

"les opinions publiques européennes connaissent une indéniable droitisation,

en particulier en matière d'immigration et de sécurité".

"L'idée selon laquelle"

on ne se sent en sécurité nulle part' recueille l'assentiment de $70 \%$ des Italiens, $65 \%$ des Espagnols, $64 \%$ des Français, contre $44 \%$ en Suisse et $43 \%$ en Allemagne." quiètent de l'organisation d'une zone militarisée entre les États-Unis et le Mexique" (France-amérique.com, 26 juin).

Dans Les Idées claires (France Culture, 8 avril), Sylvain Kahn rappelle qu'"en un mandat, l'administration Obama a expulsé deux fois plus de migrants sans papiers que l'administration Bush fils en huit ans. [Pour] Marie-Laure Basilien-Gainche: 'La cohérence de cette politique migratoire contradictoire est à chercher dans une politique normative de classement et de hiérarchisation des individus. (...) La possibilité d'obtenir des papiers en règle est offerte à ceux qui entrent dans les critères. Ceux qui y restent extérieurs sont destinés à la relégation, à la marginalité, à la précarité, aux centres de rétention et à l'expulsion."'

En Allemagne, en 2012, "966 ooo étrangers sont venus s'installer", soit une "hausse de $15 \%$ et un record en vingt ans" (Les Échos, 21 mai). "Le flux de départs est néanmoins important aussi puisque le solde net est de 369000 immigrants. Deux sur trois venaient d'Europe, surtout du sud ou de l'est." "Confrontée au vieillissement de sa population, qui privera ['Allemagne] de 6 millions d'actifs d'ici à 2030", Angela Merkel a déclaré : "Tous ceux qui peuvent contribuer à faire de l'Allemagne un pays ouvert et accueillant pour le personnel qua- lifié sont encouragés à le faire." Selon Frank-Jürgen Weise, chef de l'Agence allemande pour l'emploi, "à terme le marché du travail allemand aura besoin d'au moins 200000 arrivées nettes de personnel de l'étranger par an, afin de couvrir les besoins de main-d'œuvre" (Geopolis.francetv info.fr du 7 mai).

\section{Quand Janus ferme les portes}

En Grande-Bretagne, le Daily Telegraph du 8 avril ,inquiet, lui, de l'arrivée des Roumains et des Bulgares, demande "aux politiques, (...) non seulement [de] trouver le moyen de limiter l'immigration, mais aussi de veiller à ce que les services publics (...) soient à même d'absorber cette hausse inexorable de la population sur notre ille de plus en plus surpeuplée". Pour le site d'informations roumain Hotnews, "les informations négatives sur les Roumains génèrent un sentiment de xénophobie 'exploité par certains politiciens comme Diana James, candidate UKIP lors de l'élection législative partielle d'Eastleigh, qui a affirmé que les Roumains ont une prédilection pour la délinquance!' Elle a dû s'excuser mais a fini à la deuxième place, avec $28 \%$ des voix."

Le 8 mai, la reine a inauguré la session annuelle du Parlement de Westminster en dévoilant, au nom du gouvernement Cameron, un durcissement des lois sur l'immigration. Le Figaro (9 mai) précise que "selon les termes énoncés par la souveraine, il s'agit de 's'assurer que ce pays attire des gens qui y contribuent et repousse ceux qui ne le feront pas"'.

"Le UK Independance Party (UKIP) et une partie de la presse britannique agitent pour leur part le spectre d'une 'invasion' de Bulgares et de Roumains une fois passée l'ouverture des frontières des VingtSept aux ressortissants de ces deux pays membres, qui entrera en vigueur en janvier 2014" (Le Monde, 8 mai). Les Échos notent que "les Lib Dems et le Labour sentent aussi que ce sera un thème [l'immi- 
gration] important des prochaines élections et durcissent le ton" (10 mai).

La Suisse "a décidé de rétablir des quotas d'immigration pour l'ensemble des ressortissants de I'Union européenne" (Le Figaro, 26 avril) et, suite au référendum du 9 juin sur les conditions d'asile des étrangers, les Suisses ont approuvé "le durcissement des lois sur l'immigration" (La Croix. com, 10 juin). En Suède, "les démocrates suédois, mouvement xénophobe, pointent désormais à la troisième place dans les intentions de vote" et, selon le site du Nouvel Obs (17 avril), "les partis xénophobes ont le vent en poupe un peu partout en Scandinavie". "C'est le cas du Parti du peuple danois, membre de la dernière coalition, qui profite de l'impopularité du gouvernement de centre gauche, ou du Parti du Progrès, devenu la troisième force politique de Norvège."

En Italie, Cécile Kyenge Kashetu, première femme noire à accéder au rang de ministre, est traitée de "guenon", de "négresse", de "zouloue" et menacée de mort. "Les insultes et menaces qui me visent (...) visent en réalité tous ceux qui refusent le racisme et une société non violente", a-t-elle déclaré (Jeune Afrique, 19 juin).

Selon une enquête de l'Ifop, "les opinions publiques européennes connaissent une indéniable droitisation, en particulier en matière d'immigration et de sécurité". "L'idée selon laquelle 'on ne se sent en sécurité nulle part' recueille l'assentiment de $70 \%$ des Italiens, $65 \%$ des Espagnols, $64 \%$ des Français, contre $44 \%$ en Suisse et $43 \%$ en Allemagne. (...) Le thème 'il y a trop d'immigrés' recueille aussi l'adhésion de $83 \%$ des sondés en Belgique et $60 \%$ en Allemagne. En France elle atteint $66 \%$, en Suisse $73 \%$ et en Italie 71 \%" (Le Point.fr, 6 juin).

Le zjuin, le Guardian cite le quotidien israélien Haaretz: "L'État d'Israël tente d'expédier des dizaines de milliers de personnes venues d'Érythrée et du Soudan vers d'autres pays." "Plus de 2000 auraient été expulsées de cette manière. Des centaines auraient été expulsées depuis la prison israélienne où elles avaient été affectées." Le 20 mai, le Guardian rapportait une déclaration de Benjamin Netanyahu à propos de ces quelque 60000 migrants africains: "Ce phénomène est très grave et menace le tissu social de la société, notre sécurité nationale et notre identité nationale."

\section{L’immigré au trébuchet !}

Tandis que les politiques lèvent ou baissent le curseur qui va rejeter tel ou tel à la marge en fonction de critères électoralistes ou de tiroir-caisse, les spécialistes continuent d'interroger le rapport coûts/avantages de l'immigration. La mission sur l'innovation conduite par Jean-Luc Beylat (Alcatel) et Pierre Tambourin (Inserm) suggère d'“attirer les talents du monde" par "une politique plus agressive et plus ciblée de recherche de compétences à très haut potentiel" (Lexpansion.com, 5 avril). Selon le Cercle d'outre-Manche, un forum d'entrepreneurs franco-britanniques, "l'évolution des mentalités hexagonales est possible parce que les entreprises doivent se diversifier et attirer les talents, que la population vieillit et que le travail est le meilleur moyen d'intégrer les populations immigrées" (Les Échos, 27 mai). Pierre Milloz freine l'emballement des calculettes de Jean-Paul Gourévitch (Polémia. com, 15 avril), quand l'OCDE vante les vertus fiscales de l'immigration (Le Monde, 13 juin et Liberté Algérie, 15 juin).

Et voici qu'une nouvelle étude sur la contribution des migrants à la croissance vient d'être publiée. Elle repose sur "une évaluation quantitative des interactions entre, d'une part, le produit intérieur brut (PIB) par habitant et le taux de chômage et, d'autre part, l'immigration permanente en France métropolitaine sur la période 1994-2008" (Les Echos.fr, 7 avril). Selon les auteurs Hippolyte d'Albis, Ekrame Boubtane et Dramane Coulibaly, les immigrés stimuleraient la croissance. Entre 1994 et 2008, "le taux de migration (nombre de migrants rapporté à la population totale) augmentait de $1 \%$, alors le PIB par habitant augmentait lui d'environ 5 euros par personne et par an", soit "un gain de 300 millions d'euros" (Les Echos. 


\section{KIOSQUE}

fr, 4 avril). En distinguant les motifs et la durée du séjour, les auteurs constatent que "I'immigration rapporte, et même une immigration plutôt issue du regroupement familial" (Le Parisien.fr, 8 avril). Les femmes qui composent cette immigration familiale, "en grande partie des femmes d'Afrique noire ou subsaharienne, (...) occupent des emplois de gardes d'enfants ou de femmes de ménage et libèrent de ces contraintes domestiques des femmes autochtones plus qualifiées qui peuvent aller sur le marché du travail".

\section{Changer de logiciel?}

Sur le gril des calculettes, l'immigré(e) tremble. Pourtant, malgré le durcissement des politiques, l'exil se mondialise et le métissage renforce l'effectivité d'une conscience planétaire. La CNHI rappelle qu'un Français sur quatre est issu de l'immigration, Cameroonvoice.com du 10 mai rapporte qu'"un Canadien sur cinq (20,6 \%) est né à l'étranger" et le site Geopolis.francetvinfo. fr (7 mai) signale, lui, qu'“en 2011, près d'un Allemand sur cing et un nouveau-né sur trois est issu de l'immigration".

Les flux migratoires ne se limitent pas à une pression du Sud vers le Nord. Ainsi, "plus de 1,5 million de Syriens ont trouvé refuge" dans les pays frontaliers, en Égypte et en Afrique du Nord (Lemonde. fr 17 mai). L'Afrique est aussi une terre d'immigration. "Les mines d'or ghanéennes ne cessent d'attirer des ressortissants chinois" (Koaci.com, 13 mai) et africains (Bbc.co.uk, 10 juin). Au Maroc ou en Algérie les migrants subsahariens s'installent et les flux s'“accentuent": "En janvier 2013, ils étaient plus de 107000 à avoir trouvé refuge en Algérie" (Algerie-focus.com, 8 avril). Au Gabon, "une partie de la population expatriée, pour la grande majori- té originaire de l'Afrique de l'Ouest, vit aujourd'hui dans la clandestinité totale. Elle gagne le territoire gabonais par la route en passant par le Cameroun mais très souvent aussi par la mer en partant du Nigeria ou du Bénin (...)" (Gabonreview. com, 22 juin). En Angola, le gouvernement accuse l'immigration clandestine de menacer la stabilité (Afriquechos.ch, 27 juin) et le Congo expulse les Camerounais sans papiers (Le Jour, 21 juin). Et "l'immigration, ce n'est pas que dans un sens" (Slateafrique.com, 21 juin), "le Maroc est devenu le nouvel eldorado des Espagnols". D'après les inscriptions consulaires, ils seraient 8115 en 2012. Sans compter les 5000 Espagnols qui résident au Maroc sans être inscrits.

Côté prévisions, les Nations unies prévoient "11 milliards de Terriens en 2100, dont 3 milliards auront plus de 60 ans", et les principaux pays d'accueil des migrants seront,"sur la période 20102050, les États-Unis (1 million d'immigrés par an en moyenne), le Canada (205 000), le RoyaumeUni (172 500), l'Australie (150 000), l'Italie (131 250), la Russie (127 500), la France (106 250) et l'Espagne (102 500)" (Le Monde.fr,13 juin).

La Banque mondiale de son côté met en garde : "Si le monde se réchauffe de $2^{\circ} \mathrm{C}$, (...) cela se traduira par de vastes pénuries alimentaires, des vagues de chaleur sans précédent et des cyclones plus violents", et d'ajouter que "I'Afrique et l'Asie du SudEst en seraient les premières victimes" (Le Monde. $\mathrm{fr}, 19$ juin).

Si les faits ne suffisent pas à convaincre de la nécessité de changer de logiciel sur les migrations, peut-être que les prévisions y aideront. À quand des politiques plus efficaces, présentant l'immigration non comme une anomalie, tantôt tolérable, tantôt indésirable, mais comme la marche habituelle, consubstantielle et, parfois, inévitable des sociétés et des hommes? 